\title{
La Cruz Roja, el panamericanismo y la salud en el período de entreguerras
}

\author{
The Red Cross, Pan-Americanism and health in the \\ inter-war period
}

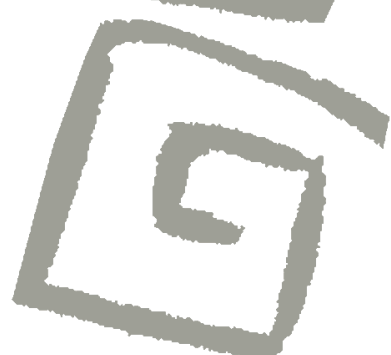

María Silvia Di Liscia ${ }^{1}$, Adriana Carlina Alvarez ${ }^{2}$

'Doctora en Geografía e Historia. Profesora Asociada, Instituto de Estudios Históricos y Sociales de La Pampa, Facultad de Ciencias Humanas, Universidad Nacional de La Pampa. Santa Rosa, Argentina. $\triangle$ (iD)

${ }^{2}$ Doctora en Historia. Docente, Centro de Estudios Históricos, Facultad de Humanidades, Universidad Nacional de Mar del Plata, Investigadora Independiente, Consejo Nacional de Investigaciones Científicas y Técnicas. Mar del Plata, Buenos Aires, Argentina.
RESUMEN La Cruz Roja, organismo internacional originalmente organizado en Europa, provocó la formación de sociedades nacionales en distintos países latinoamericanos bajo los principios humanitarios y neutrales relacionados con el tratamiento de prisioneros en épocas de conflictos bélicos. Luego de la Primera Guerra Mundial y bajo la influencia estadounidense, se impulsaron la Liga de las Sociedades de la Cruz Roja para el diseño y aplicación de acciones sanitarias y sociales en tiempos de paz. A partir de ese impulso, en la década de 1920 y 1930, se realizaron congresos panamericanos en Buenos Aires, Washington y Río de Janeiro que dispusieron una agenda coincidente con los principios de esta organización internacional que, a su vez, permitieron unificar las élites, los organismos estatales y privados de diferentes ámbitos en pos de la intervención sobre diferentes sectores sociales.

PALABRAS CLAVES Cruz Roja; Historia; Argentina.

ABSTRACT The Red Cross, an international organization originally founded in Europe, promoted in different Latin American countries the formation of national societies under the humanitarian and neutral principles in relation to the treatment of prisoners in times of armed conflicts. After the First World War and under the influence of the United States, the League of the Red Cross Societies was fostered to design and apply sanitary and social actions in times of peace. Based on that boost, different Pan-American Congresses were held in the twenties and thirties in Buenos Aires, Washington and Rio de Janeiro, which had an agenda that coincided with the principles of this international organization and, at the same time, allowed the elites and the state and private organisms of different fields to unify in pursuit of intervening in different social sectors.

KEY WORDS Red Cross; History; Argentina. 


\section{INTRODUCCIÓN}

Como sucede con muchas instituciones de larga data e importante presencia, al construir una historia de la Cruz Roja es preciso despojarla de su ropaje sagrado para que sea posible explicar su pasado. Sus principios son la humanidad, imparcialidad, neutralidad, independencia, servicio voluntario, unidad y universalidad $^{(1)}$. En un organismo de estas características, que perdura hasta la actualidad y tiene presencia mundial, es normal la existencia de historias laudatorias que la sindican como la primera institución humanitaria, prescindente de filiación religiosa y/o política, cuyo propósito era la intermediación neutral en los conflictos entre naciones ${ }^{(2)}$. Su origen se encuentra en la preocupación de un grupo de filántropos suizos por las víctimas militares de ambos bandos de las cruentas guerras del centro de Europa. Así surgió la Conferencia Internacional de Ginebra, de 1863, germen de posteriores conferencias internacionales ${ }^{(3)}$. En 1864 se firmó el Convenio de Ginebra, principal antecedente sobre derechos internacionales y base de las normas jurídicas internacionales sobre la guerra, aprobado y ratificado por numerosos gobiernos. Aunque modificado en 1949 y luego en 1977, su base general persistió(4). También en 1864 surgió en Suiza el Comité International de la CroixRouge (CICR), con cinco miembros de esa nacionalidad, que luego se incrementaron hasta superar la veintena y de diferentes países. EI CIRC no representaba a ningún Estado e, inicialmente, era una asociación privada internacional, interesada en gestionar la neutralidad para el uso de ambulancias, hospitales y transporte de heridos, así como la práctica del respeto y ayuda a los civiles.

Entre 1864 y 1919, en todo el mundo se organizaron entidades que el CIRC incorporó como Sociedades Nacionales de la Cruz Roja, a condición de que el propio gobierno al cual pertenecieran las hubiese reconocido previamente y, a la vez, que estos gobiernos estuviesen adheridos al Convenio de Ginebra antes indicado $^{(4)}$. Hacia 1870 se habían formado 24 asociaciones y dos conferencias -la de París, en 1867, y la de Berlín, en 1869- que constituyeron un espacio de intercambio para avanzar hacia una legislación internacional vinculada al humanitarismo y a la regulación de la guerra. Tanto en las publicaciones como en los encuentros se solicitó ayuda para los heridos en diferentes escenarios bélicos, se informó sobre las relaciones entre diferentes comités y los nuevos socios incorporados y se estimuló la solidaridad financiera o en especies ${ }^{(5)}$.

Luego de la Primera Guerra Mundial, la Cruz Roja tomó una nueva línea la intervención sanitaria y social, sin abandonar el militarismo, lo que implicó considerar la guerra y su preparación como una actividad normal y deseable, que tuvo una curiosa consecuencia: el florecimiento del "patriotismo de la Cruz Roja"(6). Al finalizar la conflagración, Henry Dawson, presidente del Consejo de la Cruz Roja en EEUU, convocó a diferentes países aliados de la contienda (Francia, Italia, Japón y Gran Bretaña) para formar un comité propio, presumiblemente en contraposición al ginebrino, donde EEUU no tenía injerencia, argumentando en pos de políticas para las sociedades en tiempos de paz. Con la presión de esta potencia en ascenso se fundó, en 1919, la Liga de las Sociedades Nacionales de la Cruz Roja que en poco tiempo incluyó a 30 países. El control de las Cruz Roja por parte del CICR entró en crisis y el conflicto se trasladó a distintas conferencias en los años veinte, hasta que se saldó con acuerdos en 1948.

Las conferencias internacionales fueron: París (1867), Berlín (1869), Ginebra (1884 y 1887), Roma (1892), Viena (1897), San Petesburgo (1902), Londres (1907), Washington (1912), Ginebra (1925, 1923 y 1925), La Haya (1928), Bruselas (1930), Tokio, (1934), Londres (1938) y Estocolmo (1948). A partir de entonces surgió una compleja entidad internacional, denominada "Movimiento de la Cruz Roja Internacional" que implicó a las sociedades nacionales, el CICR, las federaciones de Cruz Roja y la Media Luna Roja, la Federación de Sociedades Nacionales y a su máximo organismo, las conferencias internacionales, con capacidad de decisión y acuerdo cuyos límites son la modificación del Estatuto o del Convenio de Ginebra ${ }^{(4)}$. 
¿Por qué estudiar los congresos panamericanos de la Cruz Roja? En primer lugar, porque se entrecruzan de manera compleja la atención médica y social y el humanitarismo médico, así como la formación de profesionales y su inserción en el país y, de manera más general, las definiciones frente a conflictos internos. En segundo lugar, porque dicha entidad afirmaba una ideología supranacional laica, compartiendo las bases morales con las religiones monoteístas, pero sin definirse por alguna de ellas; justamente, se pronunciaba sobre la neutralidad, la paz y la concordancia de las naciones, en un contexto internacional en el que incidían cada vez más el nacionalismo y la violencia. Esta asociación internacional descentralizada es también de interés, dada su relación con acuerdos en los que están incluidos el ordenamiento bélico y la población civil. Finalmente, el estudio de congresos y conferencias internacionales permite observar a las comunidades de científicos, sus contactos y el intercambio que va más allá de la disciplina e incluye banquetes y excursiones turísticas, como forma de observar las prácticas sociales, más allá de la consolidación de un dominio en el campo ${ }^{(7)}$. En el caso de la Cruz Roja, no se trata específicamente de un área de la ciencia, sino de la atención a víctimas de conflictos y catástrofes, en un registro supranacional.

Ahora bien, respecto al período de análisis, cabe señalar que, en 1919, se inicia una etapa diferente para la Cruz Roja con el liderazgo de EEUU que, con relación al CICR original, plantea ciertos conflictos que se reflejan en la organización de un círculo propio de conferencias. El panamericanismo, doctrina impulsada por EEUU desde 1902, implicó un aumento de su injerencia en América Latina. En ese momento, se creó una Oficina Sanitaria Internacional, a fin de controlar las epidemias que impedían incrementar el comercio intercontinental $^{(7,8,9)}$. Entre 1911 y 1920, tanto la Primera Guerra Mundial como las intervenciones militares estadounidenses en determinados países de la región limitaron la realización de conferencias, reanudadas en 1920, cuando se relanzaron como componente esencial de la relación entre EEUU y América Latina ${ }^{(9)}$.
En la década de 1920, se asiste a un verdadero empuje conjunto que presiona, por un lado, a fortalecer el panamericanismo a través de la diplomacia y, por otro, a que las Cruz Roja latinoamericanas se afilien a la Liga de la Cruz Roja. Por entonces, se indicaban potentes razones humanitarias como mejorar la salud, prevenir las enfermedades y mitigar el sufrimiento mundial, además de otras más prácticas como la necesidad de aceitar los vínculos entre organizaciones que, en general, hacía décadas que estaban en las naciones latinoamericanas. El argumento central era la aplicación de la ciencia y el conocimiento médico en pos de una agenda de intervención, no desde el Estado, sino desde los voluntarios, quienes vigorizarían las acciones gubernamentales ${ }^{(8)}$. En 1922, la Oficina Sanitaria Internacional pasó a denominarse Panamericana. John D. Long, uno de sus directores y miembro del United States Public Health Service, indicó que estaba compuesta por siete destacados facultativos de toda América (entre los cuales figuraba un argentino, Joaquín Llambías) y entre sus funciones se contaba solicitar información sobre el estado sanitario de puertos, muelles y territorios, proporcionar auxilio en caso de epidemias contagiosas a fin de propender y facilitar el comercio entre las repúblicas y sanear puertos, bahías, sistemas de cloacas, sobre todo, destruyendo los mosquitos, a partir de un fondo que en principio era de $\cup \$ 5.000$ dólares y que luego se extendió a U\$20.000 dólares. Además, sus otras funciones eran proporcionar informes a las autoridades que lo solicitaran; "canje" (sic) de médicos, consejeros de sanidad y otros profesionales; organizar visitas a ciertas repúblicas americanas para estimular los fines médicos; publicar informes demográficos (morbimortalidad, sobre todo); y difundir, en su órgano oficial, el Boletín, toda la información ${ }^{(8)}$.

En 1924, se declaraba que todos esos fines no se habían podido cumplir, pero se avanzaba en "una inteligencia, que, es de esperarse, acabará por producir la uniformidad respecto a procedimientos en relación al comercio marítimo y métodos para dominar las enfermedades contagiosas"(8). La aprobación del Código Sanitario Panamericano, en La 
Habana, en 1924, impulsó mayor dinámica al proceso con la actuación de fundaciones y organismos estadounidenses en campañas sanitarias $^{(9,10,11)}$. Tal fue el caso de la Rockefeller Foundation y de su intervención en la lucha contra la malaria y la anquilostomiasis, entre otras enfermedades, cuya morbilidad incidía en la población rural y se consideraban un freno para la expansión productiva ${ }^{(12)}$.

A la luz de estas vinculaciones entre organismos de diferente cuño, nos interesa analizar la Primera Conferencia Panamericana de la Cruz Roja, en Buenos Aires, en 1923; la Segunda Conferencia Ilevada a cabo en Washington, en 1926, y la Tercera, en Río de Janeiro, en 1935, todas ellas en el marco tanto de la injerencia estadounidense, como de las políticas de preparación bélica de la década de 1930.

La Cruz Roja, como organismo internacional, puede analizarse a la luz del estudio de las historias conectadas, fructíferas para interpretar hechos producidos más allá de las fronteras nacionales ${ }^{(13)}$. Este estudio aporta también al conocimiento de las asociaciones sanitarias y sociales, a los estudios de género y a las conexiones entre Estado y sociedad civil, sobre todo, en el caso argentino, en un contexto mayor, en el que inciden también las instituciones transnacionales influenciadas por determinadas potencias. Si bien existen estudios sobre la enfermería y la profesionalización médica en diferentes niveles y hay avances respecto a organizaciones de la sociedad civil ${ }^{(14,15,16)}$, no han sido examinadas en profundidad las conexiones nacionales e internacionales de una asociación de este nivel.

Nuestro objetivo es el análisis de las tres primeras conferencias panamericanas realizadas en las capitales de Argentina, EEUU y Brasil en el período de entreguerras. A raíz del aspecto filantrópico, se consideran dentro del foco de estudio la conformación de grupos de interés de la Cruz Roja Argentina y la participación, en virtud de su composición de clase y género. A esta cuestión se suma la comparación con las delegaciones de otros países de América, en especial del ámbito latinoamericano, en relación con la configuración de una agenda común de debate a nivel intercontinental. Como fuente, se relevaron libros, manuales y libros institucionales y publicaciones periódicas, sobre todo la Revista de la Cruz Roja Argentina (serie completa: 1923-1974); así como el Monitor de Educación Común; el Boletín de la Oficina Sanitaria Panamericana (1924 y 1926); el órgano oficial de la Liga, The World's Health y fuentes complementarias, como American Journal of Public Health (1921) y Cruz Roja Argentina: Su programa en épocas de paz (1919).

\section{LA CRUZ ROJA EN ARGENTINA Y EL PRIMER CONGRESO PANAMERICANO}

Argentina firmó el Convenio de Ginebra en 1864 y aún sin organizar una asociación nacional, la Cruz Roja participó en la atención de heridos en la Guerra contra el Paraguay y en la lucha contra la fiebre amarilla. En 1880 se constituyó formalmente en Buenos Aires, fruto de un acuerdo entre jóvenes médicos del Círculo Médico y la Sociedad Española y estuvo presente en el auxilio a los heridos de ambos bandos en la lucha por la capitalización de Buenos Aires.

Hacia 1890, la Cruz Roja Argentina había asistido a heridos en combates e intervenido en epidemias de alcance masivo y alta morbimortalidad, como el cólera. Hasta ese momento, y dada la cantidad de médicos aglutinados, esta entidad era eslabón clave en la atención médica y, a la vez, no colisionaba con las múltiples acciones llevadas a cabo por el resto de las entidades de beneficencia en la atención a madres, huérfanos, discapacitados, enfermos psiquiátricos, por nombrar los principales conjuntos sociales afectados. Sus acciones se ampliaron hacia las "calamidades", por lo cual se desplegó un discurso conciliador hacia el resto de las entidades existentes, ubicándose como auxiliar de las tareas realizadas por las organizaciones benéficas. Uno de los primeros hechos, en este sentido, fue en 1891 las inundaciones en Córdoba, arrasada por las aguas, lo cual produjo numerosas víctimas fatales y 
pérdidas materiales. El pedido de auxilio a la Cruz Roja Argentina motivó un debate interno vinculado a estas nuevas funciones, en especial, a si el rol debía limitarse solo al auxilio de heridos en combate. La idea que primó fue que, si había sido fundada para servir en las guerras internacionales o civiles, también lo era para actuar en las grandes epidemias y catástrofes. Por lo tanto, en el caso de Córdoba se resolvió el auxilio con provisiones, así como la coordinación de su entrega ${ }^{(17)}$.

A su vez, el comité porteño estimulaba la emergencia de un subcomité de la Cruz Roja en esa provincia para evitar desplegar desde Buenos Aires toda la atención y el auxilio solicitado. En Córdoba, los delegados aunaron las dispersas colaboraciones ofrecidas tanto por las organizaciones benéficas, como por la población en general, formando un solo centro común para repartir con equidad los recursos recolectados en dinero y en especies. La Cruz Roja Argentina se puso a la cabeza en la articulación del accionar entre las entidades de beneficencia (ya fuesen estas de orden católico o étnicas) y la población afectada, sin superponerse a las otras pero con evidente interés de liderazgo ${ }^{(17)}$.

En 1890, obtuvo la autorización jurídica del Estado como Sociedad Nacional, año en que participó asistiendo a los heridos de la Revolución del Parque. A finales del siglo, las ambulancias y el personal de la Cruz Roja Argentina ya eran reconocidos como tal por la prensa, dado su papel en las contiendas internas. Tal fue el caso de las rebeliones de Santa Fe, en 1893, y Entre Ríos, en 1899, y como indicamos más arriba, en la atención a víctimas de inundaciones en la provincia de Córdoba, en 1891, y del terremoto de La Rioja, en 1894. También, la Cruz Roja Argentina hizo acopio de fondos, víveres, vendajes y otros productos para asistir a víctimas en Chile, en 1891 y en $1906^{(17)}$.

A principios de siglo, había unos 700 miembros en todo el país y filiales en distintos puntos de Buenos Aires, Córdoba, Tucumán y otras provincias. Por entonces, la entidad, de acuerdo a sus estadísticas y balances, estaba en proceso de crecimiento, indicando una diferencia positiva entre ingresos y egresos y tenía una publicación propia (Boletín de la Cruz Roja Argentina), editada desde 1891 a 1908. En 1923, se inició la Revista de la Cruz Roja Argentina bajo la dirección de Juan Monteagudo Tejedor, miembro de una influyente familia porteña, dirigida a las "enfermeras visitadoras". Los artículos indican un contacto fluido con los servicios públicos, en especial, con el Departamento Nacional de Higiene. Fue cambiando de periodicidad, director y aún de presentación gráfica y sospechamos que el impulso de su creación se debió a la selección de Buenos Aires como sede de la Primera Conferencia Panamericana.

En 1923, el Consejo Supremo de la Cruz Roja Argentina estaba presidido por Joaquín Llambías, profesional con destacados antecedentes en anatomía patológica, quien se había perfeccionado con Rudolf Virchow en Berlín y luego en el Instituto de Anatomía Patológica. Fue presidente de la Asociación Médica Argentina y profesor universitario, intendente de la Ciudad de Buenos Aires y como integrante de la Oficina Sanitaria Panamericana, uno de los expertos argentinos que en 1924 asistió a la Conferencia de La Habana. En el Consejo de la Cruz Roja Argentina participaban Raúl Ortega Belgrano, Sylla Monsegur, Pedro Lalanne, Julia Zumarán de Olmedo, Elena Green de Lanús, Elena Moutier, Roberto Doods y Adela de Pando. El Consejo General de dicho organismo estaba formado por los mismos nombres anteriores a los que se agregaban el reconocido higienista Nicolás Lozano, el general Severo Toranzo y una comisión de damas de familias notables porteñas, presidida por Lucrecia Guerrico de Ramos Mejía. Varias de las mujeres participaban en el núcleo católico, conservador y antisemita de entonces que militaba por el ejercicio de la maternidad ${ }^{(18)}$. Otras que se visualizan en las sucesivas acciones de la Cruz Roja eran ya célebres matronas, participantes de la filantropía a nivel nacional, como Dolores Lavalle de Lavalle, Mercedes Ortiz Basualdo y Mercedes Unzué de Quintana, y la Cruz Roja no era más que otra de las numerosas actividades para promover el cuidado de los sectores populares más desprotegidos y, consecuentemente, su moralización. 
Se indicaba que una de las más importantes, Guillermina César de Wilde -esposa del médico, ministro y diplomático Eduardo Wildedejaba de lado lujos y boatos para ponerse al frente de instituciones sociales ${ }^{(19)}$. A estas mujeres, la Cruz Roja seguramente les significó cierta intervención de la vida política nacional como "decisoras", en un contexto marcado por la subordinación de género ${ }^{(20)}$. La Cruz Roja, como otras actividades filantrópicas, les permitía reconocimiento y prestigio intraelitario e intromisión carente de formalidad, anclada en la pertenencia social.

En ese momento, se desplegaba el accionar, sobre todo en la Capital Federal, de la poderosa Sociedad de Beneficencia, cuyas damas, en un inicio, y otro conjunto de mujeres de clase media, posteriormente, disponían de asilos, orfanatos, hospitales, maternidades y un sinnúmero de instituciones caritativas para la atención de los más desfavorecidos, en especial, mujeres y niños. Recibía aportes de la Comisión de Asilos y Hospitales Regionales a nivel nacional y también otros apoyos públicos, y su influencia fue tal que competía exitosamente en el espacio de atención médico-social que disputaban para sí los profesionales ${ }^{(21,22)}$. Recordemos que, paralelamente a la creación de la Oficina Sanitaria Panamericana, la labor en pos de la niñez desvalida fue también preocupación de un conjunto amplio y se reflejó en la realización de Congresos Panamericanos del Niño, realizados entre 1916 y hasta $1942^{(20)}$.

Hacia 1923, la Cruz Roja Argentina comenzó a demostrar apremios económicos y necesidad de incrementar el número de socios para solventar las actividades en expansión. El crecimiento comparativo de socios en cada uno de los países parece ser una especie de "competencia": a finales del siglo XIX, y debido a la Guerra Civil, EEUU tenía solo 27.000, muy por debajo de los cientos de miles de los países europeos ${ }^{(6)}$. Sin embargo, a principios del siglo XX, el afán "patriótico" por acrecentar los asociados en ese país llevó a un desarrollo exponencial que le permitió importantes recursos económicos propios. Así, la Cruz Roja Argentina, que diez años antes estaba ufana de su patrimonio, al compararse con la nación del norte destacaba la necesidad de expandir el número de asociados como en EEUU que por entonces acreditaba 25 millones de miembros: con el aporte de un solo dólar anual se lograba reunir así una suma millonaria al margen de los aportes estatales, tema muy sensible para las organizaciones voluntarias ${ }^{(23)}$.

La Conferencia Panamericana indudablemente le permitiría a la institución local argentina reconocimiento $y$, a partir de allí, crecimiento interno. Los antecedentes de esta reunión fueron la Conferencia Internacional de Cannes de la Liga de las Sociedades de la Cruz Roja, realizada en Francia, en 1919, y la V Conferencia Panamericana Internacional, desarrollada en Santiago de Chile pocos meses antes, en 1923. De acuerdo a Llambías, la reunión de Buenos Aires no tenía solo el objetivo del acuerdo diplomático entre diferentes Estados, sino reunir en una sola estructura las instituciones hermanas para "fortificar sus organismos, uniformar sus ideas y procedimientos", de esta manera, "el mundo ha de contemplar con júbilo el sincrónico movimiento de las Naciones de América bajo el estímulo sacrosanto de sus obras de paz y amor"(24).

Los congresos panamericanos le dieron visibilidad a la Liga de las Sociedades de la Cruz Roja fuera de Europa, estrategia tejida por EEUU para ampliar su injerencia diplomática y política, Ilevándola al plano médico-social. Entre las actividades de 1921 se citaba, por ejemplo, la intervención con recursos técnicos y humanos en las campañas de malaria en España y en las definiciones de causas de mortalidad a nivel internacional a través de las normas de Bertillon ${ }^{(11)}$. Pero además, justamente el origen del Primer Congreso tiene relación con el acuerdo entre las dos organizaciones de la Cruz Roja a nivel internacional ya que, en 1923, en una reunión de carácter fundamental en Ginebra entre el Consejo de Gobernadores de la Liga y el CICR, se acordó sobre los criterios generales de la entidad humanitaria en época de guerra y de paz, asumiendo entonces que el CICR continuaría brindando su apoyo en casos bélicos y la Liga lo haría en acciones sanitarias 
y sociales, de difusión y prevención. A este acuerdo de gran relevancia asistieron, como delegados de Argentina, Nicolás Lozano, médico higienista, presidente de la Cruz Roja y uno de sus historiadores institucionales, y Eduardo Andreae, representante del CIRC y miembro del Consejo de Gobernadores de la Liga. Ambos decidieron proponer la realización de la reunión en Buenos Aires ${ }^{(25)}$.

En la Primera Conferencia participaron 110 personas. Los argentinos, como es lógico, eran los más numerosos (62 personas), presididos por el reconocido sanitarista Gregorio Araoz Alfaro, también máxima autoridad del Departamento Nacional de Higiene, principal institución nacional y organizador de un importante hito, la Conferencia Sanitaria Nacional ese mismo año. Formaban parte de la delegación argentina el Ejército, las universidades de ciudad de La Plata y Buenos Aires, el Arzobispado de Buenos Aires y muchas más instituciones. Los médicos de la Sociedad de Beneficencia destacaban en ese nutrido grupo, entre los cuales había varias luminarias nacionales como Alberto Peralta Ramos (obstetricia), Juan Garraghan (pediatría) y Adolfo Oyenard (oftalmología). Por la Sociedad Luz participó el facultativo Angel Giménez, figura notoria entre los especialistas de cuño socialista y cuyos intereses abarcaban también aspectos sociales de la medicina $^{(24)}$.

Los países americanos que enviaron delegados fueron, en primer lugar, Chile, con 23 personas -dado el antecedente de la reunión anterior-; luego Uruguay, con ocho personas, en su mayoría mujeres; y Brasil, con seis personas. Los restantes países -Bolivia, Brasil, Colombia, Costa Rica, Perú, Paraguay, Colombia, Cuba, Ecuador, Guatemala, El Salvador, Venezuela, Panamá, México y EEUU - participaron con una cantidad de entre dos y cuatro personas, en su mayoría varones. También la Liga de las Sociedades de la Cruz Roja envió representantes a la reunión porteña. Reconocidos profesionales como el juez americano John Barton Payne, diplomático y activista de la Cruz Roja en EEUU; Juan Antonio Larrosa, encargado de suministrar información sobre la recaudación de subsidios; y René Sand, uno de los dinamizadores de redes de trabajo social a nivel internacional ${ }^{(26)}$. También participó un miembro de la aristocracia, el conde Raoul de Roussy de Sales, delegado adjunto de la Liga y referente de actividades para el control de la tuberculosis en Francia junto a la Fundación Rockefeller ${ }^{(27,28)}$.

Durante quince días las actividades incluyeron visitas a centros sociales, agasajos y festejos $^{(24)}$. Esa apretada agenda se inició con un almuerzo en el Hotel Plaza, uno de los más distinguidos y lujosos edificios porteños, para luego continuar con una visita por la ciudad y un té danzante, recorridos por el Hogar y la Escuela de Enfermeras -recientemente fundados por Guillermina de Wilde-, y asilos y hospitales. En La Plata incluyó una excusión por el museo, la universidad y el Hospital de la Misericordia y también por la Colonia de Menores Abandonados de Oliveira. Las delegaciones tuvieron un banquete oficial en el Jockey Club, pasearon por el Tigre a bordo de yates y asistieron a una función de gala en el Teatro Colón ${ }^{(28)}$. Los intendentes de Buenos Aires y La Plata, el gobernador provincial y el presidente Marcelo Torcuato de Alvear (su esposa y reconocida cantante de ópera, Regina Pacini de Alvear, era la presidenta honoraria de la Conferencia) dieron la bienvenida en cada punto del trayecto a los visitantes, demostrando la jerarquía del evento. Así aparece el doble papel de la conferencia: por un lado, las reuniones técnicas y visitas a centros sociales y, por otro, los bailes, veladas teatrales, banquetes y galas propios de los encuentros entre las élites.

La conferencia inaugural abierta por el Ministro de Relaciones Exteriores, Angel GaIlardo, puso sobre el tapete las condiciones de Argentina como anfitriona de estos encuentros, ya que a partir de entonces las jóvenes naciones de América pasaban de ser el escenario de luchas fratricidas a ejemplarizar la paz y la confraternidad ${ }^{(24)}$. Las preocupaciones de los delegados giraron en torno de la lucha contra el alcoholismo y las drogas lesivas, la tuberculosis, las enfermedades venéreas, el paludismo, la anquilostomiasis y otras epidemias. 
En relación con la atención médica se debatió sobre la organización de las enfermeras y la protección de la infancia. Esta nutrida comisión, coordinada por el entonces director del Departamento Nacional de Higiene, Gregorio Araoz Alfaro y Guillermina de Wilde, concentró tanto a los médicos referentes de la Sociedad de Beneficencia como a las autoridades de la Cruz Roja Argentina. Así, entre los participantes se encuentra a Llambías, Peralta Ramos, Garraghan, Oyenart y también a visitantes extranjeros, como René Sand y Long $^{(23)}$.

La conferencia resolvió sobre la organización de sociedades nacionales, la cooperación entre estas y varias instituciones de beneficencia y de higiene, el desarrollo de la Cruz Roja de la Juventud, la celebración de conferencias panamericanas en plazos no menores de dos años ni mayores de cuatro, la organización de la conferencia en Washington y la determinación de la fecha de futuras reuniones, con acuerdo entre la sociedad nacional y la Liga de Sociedades de la Cruz Roja. También resolvió sobre la reorganización del Consejo de Gobernadores de la Liga, la educación de enfermeras, la colaboración a la protección a los niños, la intervención en la educación higiénico-popular y en campañas de sanidad pública ${ }^{(29)}$. Merece destacarse el aporte femenino mayoritario de la delegación uruguaya. La memoria sobre la fundación de un sanatorio materno-infantil en Montevideo de Matilde Regalía de Roosen exponía el contexto en el que la filantropía femenina escalaba a pasos agigantados los espacios masculinos a pesar de su limitación en la atención profesional a los sectores populares ${ }^{(30)}$.

Al mismo tiempo que se desarrolló la conferencia, la Cruz Roja organizó una Exposición de Higiene, donde la entidad local desplegó las ambulancias, camillas y el equipamiento sanitario moderno para admiración del público. A esa exposición se plegaron otras instituciones, como la Sociedad de Beneficencia de la Capital Federal con juegos infantiles y un novedoso sistema de destete, así como la Sociedad de Cantinas Maternales, fundada para proteger a mujeres y niños débiles a través de comedores en distintos puntos del ámbito porteño para distribuir alimentos entre otras estrategias sociales ${ }^{(31)}$.

Por lo tanto, la Primera Conferencia permitió demostrar de muchas y variadas formas los avances institucionales, la preparación técnica de cuadros y el entusiasmo de las élites vinculadas sobre todo con relación a acciones médico-sociales. De esta manera, se favoreció el movimiento organizado por EEUU y su integración en un contexto mayor con otras entidades ${ }^{(32)}$.

La repercusión del evento puede observarse a través de la publicación oficial de la Oficina Sanitaria Panamericana, que reprodujo el discurso de John Long en Buenos Aires. En este caso, además de las loas a la unión de las repúblicas "hermanas", el funcionario estadounidense y miembro director de las Ligas, resaltó el peso de las "organizaciones voluntarias de salubridad no para que ocupen el lugar del gobierno sino para que reglamenten y den vigor a su trabajo", sobre todo, en virtud de la difusión, la formación de la opinión pública y la aprobación de gastos vinculados a la salubridad. Long enfatizó las ventajas que tendría en América Latina (considerando una diferencia substancial con las otras organizaciones hermanas, las del norte), financiar obras de saneamiento e higiene pública. De esta manera, se limitaría la alta mortalidad general e infantil, que escalaba cifras escalofriantes, y se aumentaría la esperanza de vida $^{(8)}$.

Hacia 1924, René Sand daba cuenta de la miríada de organismos transnacionales que estructuraban el panorama de la atención y control social de las multitudes, entre las cuales se intentaba colocar a la Liga como coordinadora de una tarea de enorme magnitud $y$, a la vez, a la salud como el vector fundamental de integración global ${ }^{(33)}$. Se trataba, además, de un asunto que iba a chocar, en algún sentido, con los esfuerzos por organizar sistemas sanitarios públicos o políticas de alcance nacional, esbozados en algunos países como, justamente, Argentina y Brasil. 


\section{La segunda conferencia y el papel de EEUU}

La Segunda Conferencia, organizada en Washington, en $1926^{(34)}$, volvería sobre algunas de estas conclusiones, por lo cual el esquema de reuniones iniciado en Buenos Aires es un indicador de la consolidación de un tipo especial de vinculación que aunaba la preocupación social y humanitaria en diferentes sectores de las élites. El médico Clemente Trejo indicaba que, tanto en la Cruz Roja como en otras entidades, era necesario desarrollar escuelas de enfermeras y enfatizaba la atención en consultorios gratuitos para los pobres y obreros, "sin distinción de patria ni de credo" y "la fundación de maternidades, refugios para niños y mujeres abandonadas sin averiguar su nombre ni estado civil"(35). A la vez, estos eventos demostraban el liderazgo de EEUU en un contexto latinoamericano de expansión de las inversiones empresariales e influencia financiera, por un lado, y de interés cultural, por el otro ${ }^{(36,37)}$.

En 1925, se iniciaron los contactos en Buenos Aires para la organización de la Segunda Conferencia Panamericana. Los miembros argentinos destacaron que fueron invitados por el embajador de EEUU en Argentina, Peter Jay; por el director general de la Unión Panamericana, Leo S. Rowe; y por el mismo presidente de ese país, Calvin Coo$\operatorname{lidg}^{(38)}$. Este mandatario, miembro del Partido Republicano que gobernó entre 1923 y 1929, se hizo eco de leyes en contra de la expansión impositiva y promovió un control más severo del ingreso de inmigrantes. Había apoyado la Cruz Roja como vicepresidente y funcionario en Massachusetts y destacó en diversos discursos su loable actividad en EEUU, así como la participación de voluntarios y las donaciones en metálico y pertrechos para paliar la inundación del Río Misisipi, en 1920, y asistir a los soldados durante la Primera Guerra Mundial. En esta década se expandió el número de asociados de la Cruz Roja en EEUU de una forma exponencial: en 1926, se ufanaban de tener 3.012.055 miembros, 3.537 comités en todo el país y 5.500 .000 junior members ${ }^{(39)}$. Habiendo logrado ese nivel de adhesión, que implicaba además recursos millonarios anuales captados a través del pago anual, resultaba poco práctico desaprovechar el entusiasmo y desmovilizar a los socios; antes bien, el impulso dado a la salud pública significaría prestar atención a nuevos e ingentes problemas posteriores a la guerra, de orden social y médico ${ }^{(40)}$.

En este contexto de auge no sorprende que estuviesen invitados a la conferencia, además de las sociedades nacionales de los países americanos, la Unión Panamericana, la Fundación Rockefeller, el Comité Internacional de Enfermeras, la Oficina Internacional del Trabajo, la Unión Internacional de Socorros Mutuos, la Orden Soberana de los Caballeros de Malta y la Unión contra el Peligro Venéreo y la Tuberculosis, entre otros organismos de referencia vinculados a la circulación de personas, bienes y a la sanidad internacional ${ }^{(38)}$. La conferencia era un espacio de importancia para que EEUU ajustara los lazos con las naciones americanas hacia las cuales se desplegaba una estrategia de seducción en relación con la organización filantrópica que, a su vez, permitiera separar los tradicionales vínculos con el "Viejo Mundo". Este proceso se une a la expansión económica estadounidense, a través de compromisos financieros y de empresas de esa nacionalidad que buscaban nuevos mercados en América en la década de 1920.

Volviendo a la Conferencia Panamericana de 1926, la Liga de las Sociedades propuso que todas enviaran una memoria y que funcionarían cinco comisiones: 1) Estudios sobre métodos empleados en materia de organización y propaganda, 2) La acción de socorro de la Cruz Roja y personal; 3) El mejoramiento de la salud; 4) Preparación y acción de enfermeros, y 5) La Cruz Roja de la juventud(38).

En ese momento, la Cruz Roja Argentina estaba integrada por personas designadas por el presidente de la nación, quien colocaba en ese lugar preferentemente a profesionales, tanto miembros de las Fuerzas Armadas como del Departamento Nacional de Higiene. En 1926, la presidía el director general de Sanidad del Ejército, el médico Julio Garino como delegado del Poder Ejecutivo Nacional y su 
vice era también médico, Abel Zubizarreta, director de la Asistencia Pública. Como secretario, se desempeñó también el médico Gregorio Tejerino, a su vez jefe de Sanidad de la Armada; además de un secretario (Pedro Lalanne), un tesorero (Horacio Beccar Varela); entre los vocales se nombró a Gregorio Araoz Alfaro, quien era presidente del Departamento Nacional de Higiene, al médico Felipe Justo de la Facultad de Medicina y como delegados del Poder Ejecutivo a GuiIlermina de Wilde y a Justa Varela de Lainez, además de invitar al vicealmirante Vicente E. Montes y otros dos facultativos. En este listado, que continuó sin cambios por dos años, se agregó a la directora de hogares y enfermas, Cecilia Grierson, primera médica argentina, al director de Administración, general Severo Toranso, al director de Escuelas de Enfermeros y estaciones sanitarias, al médico Nicolás Lozano, y al director de la Cruz Roja de la Juventud, profesor Próspero Allemandi ${ }^{(41)}$. En junio se modificó en parte el staff, incluyendo a Angel Roffo como vicepresidente (médico referente sobre la atención del cáncer en Argentina) y al facultativo Angel Giménez como prosecretario, vinculado a la estadística médica y los problemas sociales.

En la Cruz Roja de la Juventud, se reemplazó al representante por el profesor Julio Picarel, inspector escolar y miembro del Consejo Nacional de Educación, organismo central en la definición de políticas educativas. También se modificaron los vocales ${ }^{(42)}$. Así, progresivamente, tanto los organismos como los integrantes de la Cruz Roja Argentina tenían relación directa con servicios públicos de salud, tanto de atención, prevención como formación. Llama la atención la conformación de la Cruz Roja de la Juventud, que se había abierto poco tiempo antes, quedando a cargo de un docente en lugar de un médico. Este papel preponderante tenía relación con el propósito de este espacio con relación a las nuevas generaciones. Hacia 1924, una publicación dependiente del Consejo Nacional de Educación mencionaba la intención de la Cruz Roja de formar a los más pequeños, atendiendo a su vocación de ayuda en tiempos de paz ${ }^{(43)}$.
Volviendo a la conferencia, los argentinos asistentes fueron Gregorio Araoz Alfaro -quien además debía presidir en París la Comisión para el Código Sanitario- y Guillermina de Wilde, quien "generosamente" había dejado de lado un viaje de placer a Europa. También, participaron como delegados el presidente y los secretarios de la Cruz Roja Argentina. En la conferencia se puntualizó la nueva agenda, vinculada con asuntos sanitarios y sociales, que incluía la cooperación entre autoridades sanitarias para la lucha contra enfermedades infecciosas y sociales, como la tuberculosis, sífilis y paludismo. En general, se asumía un programa de corte maternalista, con la protección de mujeres y niños a través de la capacitación de médicos y auxiliares. La Cruz Roja también incluyó servicios de asistencia pública y primeros auxilios en pueblos que no los tuviesen; tal situación implicaría el apoyo y hasta el cuasi reemplazo a la sanidad estatal cuando la expansión de la medicalización era parte de un proyecto aún no realizado en América Latina ${ }^{(44)}$.

Los países representados fueron además de EEUU, Argentina, Bolivia, Brasil, Canadá, Colombia, Costa Rica, Cuba, Chile, Ecuador, El Salvador, Guatemala, Paraguay, Uruguay y Venezuela. Además, estuvieron presentes en carácter de observadores ocho países no americanos y de seis organizaciones internacionales $^{(34)}$. Argentina y EEUU, Brasil, Chile, México, Cuba, Venezuela, Puerto Rico y Canadá tuvieron como representantes a los presidentes respectivos de sus sociedades. El programa dibujado por la Segunda Conferencia era claramente el de los "tiempos de paz", enfatizado por la Liga. Sus conclusiones fueron mucho más específicas que las de la Primera Conferencia: la I Comisión indicó que la integración de las sociedades debía incluir al Ejército, la Marina y al Departamento de Higiene, sin diferencias de "sexo, culto u opinión pública", para buscar entre los asistentes de los órganos de decisión el principio de "solidaridad moral". Se insistía sobre el establecimiento de la Oficina Sanitaria Panamericana, que coordinaría las acciones de la Cruz Roja en todo el continente y utilizar folletos y película, además de la 
revista de la Liga, The World's Health, para difundir su labor ${ }^{(45)}$.

En esta comisión se aceptó con el voto de los delegados la propuesta de Nicolás Lozano sobre la necesidad de que la Cruz Roja generara un centro coordinador de acciones con criterio científico y una oficina estadística paralela a los organismos nacionales. La Cruz Roja podía desempeñar un papel complementario o supletorio en los países sin ese servicio y, en tal caso, utilizar las visitadoras sociales y enfermeras para relevar la información ${ }^{(46)}$.

Este punto reviste un central interés por su referencia a las organizaciones de beneficencia argentina y un conflicto entre médicos y damas de la Sociedad de Beneficencia de más larga data. A finales del siglo XIX se enfrentaron ambos grupos, entre los cuales se contaba a las nuevas generaciones de higienistas (como Wilde, José María Ramos Mejía y Guillermo Rawson) en pos de diferentes formas de administrar las instituciones sanitarias y sociales más relevantes de la Capital Federal. Recordemos que varios de esos facultativos fueron parte del sector que en 1880 habían pujado (y perdido) en la primera fundación de la Cruz Roja Argentina frente a la Sociedad Española de Beneficencia ${ }^{(17)}$.

La problemática ahora era la oposición entre la formación técnica adecuada de personal femenino auxiliar (enfermeras), al margen de los logros de la Cruz Roja Argentina, que contaba con decenas de escuelas y personal pero, según indicaba uno de sus críticos, que requería mayor profesionalidad y/o formalidad en sus estudios. Lozano indicaba que en los países en guerra hubo necesidad de formar con rapidez personal auxiliar, pero esa no era la situación en Argentina, donde convenía tener menos mujeres capacitadas, pero de manera más profesional. Tal fue la razón por la cual en esos años se convino que las escuelas de enfermeras fundadas por GuiIlermina Wilde, muy publicitadas en 1923, pasasen a ser de Samaritanas. Según Lozano, por entonces Director de Escuelas de Enfermeras y Estaciones sanitarias y luego titular de la Cruz Roja Argentina, la filantropía había perdido su carácter instintivo y sentimental para hacerse científica y adquirir eficacia ${ }^{(25)}$.

La II Comisión insistió sobre la organización de la Cruz Roja en épocas de guerra, apuntando a la obligación de prestar socorro en caso de desastres. En tal sentido, la Liga también se hacía eco de organizar la ayuda y de brindar información científica. La III Comisión asumió una agenda claramente maternalista y de protección social con relación a la atención en tiempos de paz. Se trató la formación de médicos, visitadoras y enfermeras, indicando una clara subordinación de género y dos aspectos claves de la agenda social de protección más novedosos: la atención materno-infantil y el certificado médico prenupcial, bajo el subtítulo de "eugenesia". También se incluyeron temas clásicos de la higiene social, propios de problemáticas anteriores, como la vivienda y la salud de los obreros, con un énfasis puesto en la "enseñanza popular", es decir, en el fortalecimiento de la medicalización de los sectores populares y, a la vez, de intervención en las jurisdicciones donde los Estados americanos tenían escasa presencia $^{(45)}$. Todos estos tópicos ya estaban presentes en muchos de los países que formaron parte de la conferencia y con cierto desarrollo nacional, en el caso de Argentina y Brasil, como indicamos y también Uruguay y Chile.

La IV Comisión avanzó sobre un aspecto original respecto de las otras reuniones: la organización de la Cruz Roja de la Juventud, integrando a este suborganismo a los sistemas educativos de los países participantes. La $\mathrm{V}$ Comisión incorporó los principios de la Conferencia Internacional de 1921 en caso de guerra civil, declarando nuevamente la neutralidad como un principio de las sociedades en toda América. Se destacó la protección a la infancia, prevención de la ceguera, salud escolar, enseñanza popular de la higiene, puestos de enfermeras en regiones poco pobladas, habitaciones obreras, higiene industrial y nutrición ${ }^{(45)}$. Tal como en el caso de la Primera Conferencia, la Oficina Sanitaria Panamericana se hizo eco de los afanes de las Ligas de la Cruz Roja, amplificando esta información por la prensa oficial ${ }^{(46)}$. 


\section{Tercera Conferencia en Río de Janeiro}

A pesar de las bienintencionadas conclusiones anteriores, la periodicidad de las conferencias panamericanas no se mantuvo de acuerdo a la decisión original (entre dos y cuatro años), ya que la Tercera Conferencia se realizó nueve años después, en Río de Janeiro en 1935 y la Cuarta en Santiago de Chile en 1940. Ya por entonces formaban parte de las delegaciones países africanos y asiáticos y se enviaba a observadores de instituciones internacionales ${ }^{(47,48,34)}$.

En la década de 1930, el contexto económico era diferente ${ }^{(49)}$. A la crisis y el repliegue del comercio internacional se sumaron el creciente nacionalismo y la militarización que, en el caso latinoamericano, implicaría frecuentes rupturas autoritarias de las difíciles construcciones de anteriores regímenes democráticos (y no tan democráticos). La crisis había impactado a nivel social en diferentes naciones; a mediados de la década se mencionaba en la publicación oficial argentina que la miseria provocada por el paro forzoso había obligado a las sociedades nacionales a multiplicar la red de sus obras sociales, imponiendo nuevas cargas, pero demostrando las posibilidades de adaptación de la entidad ${ }^{(50)}$. La expansión de las sociedades nacionales era notable: hacia 1933, tanto la Cruz Roja como la Media Luna Roja disponían de 25.900.000 miembros (13.300.000 adultos y 12.600 .000 juniors) y un año después había 28.680.000 miembros, casi 3 millones más (14.310.000 adultos y 14.290 .000 juniors) ${ }^{(50)}$.

La crisis económica y social tuvo un correlato político. En diversos países europeos surgieron o se consolidaron experiencias totalitarias y/o fascistas y en América Latina, hubo una mayor influencia nacionalista, tanto de cuño populista como autoritaria. En 1930, Brasil registró un movimiento militar que llevó al poder a Getulio Vargas y produjo un cambio significativo en el sistema político. La Cruz Vermelha se había establecido en 1907 con el auspicio argentino, aunque en Brasil la historia oficial de la entidad se inicia con posterioridad $^{(17)}$. La delegación brasileña asistió a la Primera Conferencia en Buenos Aires y en la Segunda tuvo intenso contacto con la delegación argentina, compartiendo eventos y actividades sociales. Ambas sociedades tenían en común la participación de miembros de las Fuerzas Armadas entre sus principales dirigentes ${ }^{(45)}$.

La Tercera Conferencia se realizó bajo el auspicio del Instituto Oswaldo Cruz, ámbito de trabajo de uno de los prohombres brasileños y héroe indiscutido del panteón científico. Según el subsecretario general de la Liga, Juan Antonio R. Larrosa, la Cruz Vermelha había sido fundada por Oswaldo Cruz con las contribuciones de Ana Nery, del mariscal Ferreira do Amaral y del médico Getulio Dos Santos. En el nutrido programa se mencionaron temas ya tratados a las reuniones anteriores (relación entre sociedades nacionales y gobiernos, higiene y asistencia en hospitales y dispensarios, maternidad e infancia, y lucha contra enfermedades sociales, como la tuberculosis y las venéreas) y se agregaron nuevos tópicos: acción sanitaria de la Cruz Roja en las carreteras y la aviación sanitaria, higiene rural y en la Marina Mercante, atención de enfermedades tropicales y el cáncer, además del "servicio social, sobre todo en lo que se refiere al paro forzoso" ${ }^{\prime \prime 51)}$. En esta agenda persistía la ayuda en tiempos de guerra ${ }^{(52,53)}$ pero ya es posible entrever que la Cruz Roja no era aquí la única sino que estaba en competencia con otras de igual tenor, como el Rotary Club. Esta asociación tenía como finalidad la coordinación de diversos profesionales, surgió a principios del siglo XX en EEUU y luego se expandió a diferentes países, entre los cuales estaban los latinoamericanos, participando en campañas de vacunación y lucha antipoliomelítica ${ }^{(54)}$.

En el marco de la Tercera Conferencia, el CICR dio a conocer el encuadre de la entidad dentro de los servicios de sanidad del Ejército, insistiendo en la participación de este organismo a través de comisiones mixtas que incluyeran militares y miembros de las sociedades nacionales. El ejemplo era el de la Guerra del Chaco entre Paraguay y Bolivia, donde los voluntarios del Rotary Club habían estado más presentes que la Cruz Roja a pesar de que el Convenio de Ginebra y la IX 
Conferencia Internacional de la Cruz Roja organizada en Washington, en 1912, dejaban en claro la vigencia del "Código de prisioneros de guerra" y la atención de combatientes y excombatientes que involucraba la sanidad militar y, en consecuencia, a la Cruz Roja. Sobre los terribles efectos de la guerra y la participación de miembros argentinos de la entidad, ilustra la crónica del médico Carlos de Sanctis ${ }^{(55)}$. El CICR indicaba que, en la conflagración, ambos bandos utilizaron las técnicas más modernas para la atención de heridos; por ejemplo, los bolivianos hicieron uso de aeroplanos sanitarios y en Paraguay hubo vapores-hospitales. Sin embargo, la ayuda de la Cruz Roja debió ser insuficiente, dada la abrumadora cantidad de víctimas de ambos países ${ }^{(56)}$.

Una de las resoluciones de la Tercera Conferencia incluía "Conservar, por un lado, la salud en su más amplio sentido y, por otro, la salvaguarda de la civilización en su concepto más amplio y cabal"(57). Los delegados de los países americanos se felicitaban por la paz que reinaba en las Américas, entendiendo que la guerra engendraba más guerra y dificultades para las actividades de la Cruz Roja. Se enfatizaba que en los juniors debían engendrarse los sentimientos de "patria y humanidad" que lejos de contraponerse, otorgaban "aspiraciones e intereses fundamentales que coordinan el bienestar y el progreso de las naciones", para la "convivencia de la civilización"(57). Picarel, delegado argentino y miembro del Consejo Nacional de Educación, indicó en su exposición que la "conciencia pacificadora" no estaba opuesta a la patriótica, y que justamente los niños debían formarse en una escuela sin prejuicios históricos, bajo el signo de la fraternidad humana, porque la patria y la humanidad estaban unidas ${ }^{(58)}$. Este docente, a cargo del desarrollo de la Cruz Roja de la juventud en Argentina, fue también el autor de la letra del Himno de la Cruz Roja, con música del reconocido compositor Carlos López Buchardo. El objetivo de la Cruz Roja para los más pequeños era fortalecer una conciencia ciudadana a través de la Constitución Nacional y de otras formas de difusión para la vida democrática con cierta repulsa hacia el nacionalismo extremo. La salvaguarda de la paz en la efervescencia nacionalista y militarista de los años treinta planteaba un desafío para esta institución y el hecho de que se argumentara también en pos de la civilización sin duda era una forma de solución elegante, dado que la guerra se legitimaba para la defensa de una forma de vida occidental y moderna. Tal visión era coherente con la filantropía que participaba en la Cruz Roja a nivel general y no entraba tampoco en contradicción con los principios panamericanos propendidos en la Liga, pero sin duda significaba un contrapunto respecto del creciente nacionalismo en distintos puntos del globo.

\section{CONCLUSIONES}

Este análisis de la Cruz Roja a través de los tres primeros congresos panamericanos, centrando la mirada en el caso argentino, da cuenta de una miríada de cuestiones. En primer lugar, se observa el intercambio entre colegas con diferente nivel de experiencia en la labor llevada a cabo en instituciones sociales privadas y/o públicas, marcando una direccionalidad de las tareas reformistas sobre la base de una mirada común a los sujetos enfermos, los que supuestamente requerían atención especial en orfanatorios, cárceles y asilos, y cuyas desventajas, dolencias o acciones compelían a una acción más directa. Esos cuadros técnicos, conformados por profesionales médicos, sobre todo, insistían en asumir una tarea cada vez mayor en pos de la medicalización de los sectores populares, incorporados ahora en épocas de paz.

En segundo lugar, es preciso reflexionar a la luz de estos congresos sobre la sociabilidad de las élites y de los técnicos, es decir, de los filántropos y voluntarios de diferentes provincias y asociaciones de diversos países, forjando a nivel internacional una comunidad de intereses con una base humanitaria en común, más allá de las distinciones nacionales. Las tres primeras conferencias panamericanas supusieron la formación de un 
núcleo filantrópico transnacional orientado a objetivos propios de esta entidad y a la conformación de las propias necesidades intraelitarias y profesionales. Las reuniones en las comisiones y las visitas a instituciones sociales en Buenos Aires, Washington y Río de Janeiro fueron también momentos para aunar los lazos y comunicar experiencias, transmitidas de manera "natural" a los ámbitos y espacios de sociabilidad notabiliaria. Se detecta cierta modificación en la composición de los participantes en la Primera Conferencia respecto de la Tercera: Ios más de diez años entre una y otra introdujeron ciertas alteraciones con un avance de la legitimidad de los militares dentro de los grupos más acomodados, además de mayor peso para las mujeres. Las conferencias pueden haber sido también un espacio para confraternización en tiempos de paz para los "filántropos" seriales, concepto irónico pero certero respecto a personas de clase alta que participaban de manera sistemática en diversas actividades para el cuidado y la protección de los sectores más desfavorecidos, presentes tanto en la Cruz Roja como en otros organismos confesionales y no confesionales de los diversos países americanos.

Este análisis nos presenta un escenario donde la Cruz Roja no es la única institución que está en proceso de expansión, sino quizás sí la más importante, habida cuenta de los millones de personas que formaban parte en todo el mundo y de su notable incremento anual a partir de 1920. En Argentina, la Sociedad de Beneficencia era un socio difícil de soslayar. Que se llamara a participar a varios de sus miembros en sus distintas actividades integraría la explicación plantada con anterioridad y, a la vez, el deseo de fortalecer los propios objetivos del humanitarismo médico. La competencia con otras asociaciones permite tomar el pulso de la participación creciente de otros sectores y no solo de la alta filantropía, en la preocupación en la guerra y la paz. Tenemos un conocimiento mayor sobre algunas de estas agencias (como la Rockefeller Foundation, integrada a la Oficina Sanitaria Panamericana), pero menos sobre otras, como el Rotary Club. La estrategia de desarrollo de la Cruz Roja fue diferente y seguramente se vincule a instituciones como el scoutismo, de importante influencia en el siglo $X X^{(59)}$. Al incorporar en sus filas a jóvenes miembros en plena escolarización que asistían a escuelas públicas, la Cruz Roja se expandía horizontalmente, no solo a nivel social, sino también etario.

En esta colaboración y competencia entre las distintas asociaciones no se descarta que incidiera la situación de los montos recaudados en concepto de aporte de los voluntarios o de ayudas del erario público, tema sensible y que requeriría un examen más profundo. Para las Cruz Roja latinoamericanas, aumentar el número de socios representaba sostener las asociaciones propias con cuotas anuales, contar con mayor número de personas comprometidas y aumentar la autonomía financiera. A la vez, eso significaría menor posibilidad de involucrarse con los requerimientos de los poderes estatales, cuestión de relevancia si se deseaba un ejercicio realmente independiente que asegurara la neutralidad. Tal situación se verificaba en EEUU, quien había aumentado su número de socios haciéndose un espacio importante entre las asociaciones a nivel internacional y también, obteniendo un presupuesto significativo. Ese modelo, difícil quizás de realizarse en los países latinoamericanos, impregnaba a las sociedades nacionales de la Cruz Roja y al menos, en el caso argentino, implicaba una diferencia singular respecto de otras organizaciones benéficas.

En tercer lugar y de manera general, se abrió el camino formal para la participación femenina en las sociedades nacionales, aunque las mujeres (de clase alta, sobre todo), ya estaban insertas entre el staff de los organismos a cargo. Su papel fue mucho más importante en los años siguientes, lo cual no necesariamente indica un proceso hacia la igualdad de género sino más bien una asimilación de las labores de paz a las tareas femeninas "por naturaleza", dejando de lado las de la guerra al honor y la valentía masculinas. Tal camino parece haber sido recorrido con anterioridad por las mujeres uruguayas, que en la Primera Conferencia llevaron una nutrida delegación femenina y puede relacionarse con los roles 
profesionales femeninos a raíz de los avances de políticas sociales -también maternalistas- del gobierno de José Batlle y Ordóñez, en Uruguay, pero sin duda es un aspecto a profundizar para toda América Latina en la relación de las mujeres y el Estado, cuando estas no tenían participación plena ante una ciudadanía restringida y restrictiva. La permanente insistencia al ejercicio en las políticas públicas para proteger a madres e hijos amplificó la tarea de las voluntarias de las élites a los sectores medios, sobreponiéndoles las adjetivaciones de técnica, eficaz y moderna a las acciones filantrópicas tradicionales.

En cuarto lugar, es notable la incidencia de EEUU en la organización de nuevas actividades que permitieran una injerencia mayor en asuntos no estrictamente bélicos en el continente americano, desplazando en el imaginario cultural y social a los países europeos. La agenda de la paz, coincidente con un programa sanitario y social panamericano, estaba a cargo de EEUU y, como se trataba de aspectos en desarrollo en varios países de América Latina, no es de sorprender que haya sido de amplia aceptación. Tal cuestión, esbozada en la Primera Conferencia, en la medida que se trataba de una propuesta de la Liga de las Sociedades de la Cruz Roja, se profundizó en la Segunda y la Tercera. En el caso de Argentina y Brasil, algunos delegados aceptaron que hospitales, médicos $y$, sobre todo, equipos técnicos y personal auxiliar de la Cruz Roja estuvieran a tono no solo con la atención de heridos en las contiendas fratricidas o conflictos bélicos externos, sino que dispusieran para "el combate" de las enfermedades crónicas, epidémicas y endémicas detectadas como problemáticas pero, a la vez, difíciles y onerosas de eliminar. La preocupación por la atención materno-infantil fue otro aspecto singular, que en la década de 1930 y a partir de la crisis económica, se incorporó como parte de la cuestión social (trabajo, vivienda y derechos laborales, entre otros aspectos). Es interesante observar que, a partir de 1923, las conferencias subsiguientes perdieron la denominación de "internacional" que tenían originalmente, resguardando la de "panamericana". Un aspecto a destacar es que el accionar vinculado a la salud fue anterior al acuerdo del Código sanitario de 1924, por lo tanto, se trata de un antecedente relevante que da cuenta de la influencia panamericana en virtud de esta agencia internacional, a través de la Liga de las Sociedades de la Cruz Roja, liderada a su vez por EEUU. Los nexos con la Oficina Sanitaria Panamericana permiten además adentrarnos en las perspectivas de esta entidad, quien bregaba por mayor intervención económica en América Latina. Podríamos pensar que incentivar a voluntarios a las acciones médico-sociales, difundiendo los ideales higiénicos y sanitarios entre los Estados, sería una especie de "caballo de Troya" utilizado por el imperialismo estadounidense para un mayor ejercicio de la dominación económica (como privilegiar los gastos en infraestructura para saneamiento urbano y mejoramiento de puertos), pero a diferencia de ser un instrumento de traición, se manifestaba a cara descubierta y como forma de presión para lograr un bien mayor, superador de las diferencias y facciones.

En quinto lugar, las conferencias visualizaron una tecnología de punta en la atención médico-sanitaria, a la luz de la integración de transportes y comunicaciones modernas. La fascinación por los aeroplanos está en esta clave, ya que en la medida que abandonaban los cielos de las batallas, se tornaban instrumentos útiles para el transporte de enfermos o de vituallas en lugares inaccesibles. También era el caso de las ambulancias, que no solo debían encontrarse en previsión de futuros combatientes heridos, sino para auxiliar en catástrofes o epidemias. La profusión de información sobre enfermeras, visitadoras y asistentes en cada reunión daba pie para fundamentar los principios de la Cruz Roja, forjados en la abnegación y el sacrificio, pero ahora también en el conocimiento científico de los auxiliares de los médicos (en su mayoría, también mujeres).

En sexto lugar, y vinculado con lo anterior, tanto los medios técnicos como humanos brindados por la Cruz Roja y debatidos en las conferencias sugerían las posibilidades de medicalización en áreas donde los Estados americanos y mucho más, los que 
estaban al sur de EEUU, tenían escasa presencia, aunque deseos de expansión. ¿Se trataba entonces de un reemplazo y privatización de las funciones públicas respecto a la salud? Podríamos responder afirmativamente ya que las sociedades nacionales de la Cruz Roja, formadas en consonancia con el Convenio de Ginebra, para funcionar requerían de una buena relación o al menos, de ciertos contactos políticos. En el caso argentino, la rispidez en la gestación inicial entre Cruz Roja y Poder Ejecutivo se fue limando y gran parte de su composición dependía de la designación presidencial, tanto fueran militares como funcionarios del Departamento Nacional de Higiene. El acuerdo con estos dos organismos implicaba para el Estado extender su brazo a otras áreas y espacios públicos del interior, donde había muy escasa o nula presencia ${ }^{(60)}$. En países donde la formación de los núcleos de atención sanitaria era aún menor, como en Paraguay, la Cruz Roja proporcionaba cierta base para políticas vinculadas con la higiene, aún de manera incipiente. En momentos de crisis, la colaboración entre organismos sanitarios y distintas fuerzas del ejército permitiría avanzar a la Cruz Roja como una asociación paraestatal y al Estado, paralelamente, promover acciones en espacios donde había pocos avances.

Pero también cabe una negativa que modere la afirmación anterior, apuntando a las preocupaciones propias de esta agencia. La internacionalización llevaba implícita una neutralidad, real o ficticia, que debía mantenerse formalmente ante el CICR y la Liga. En las acciones independientes, las sociedades nacionales fortalecían su identidad y la celosa defensa de las insignias ante su manipulación o uso indebido o la creación de un himno propio, en el caso argentino, da cuenta de este fervor por hermanarse con otras, allende las fronteras. Las sociedades de la Cruz Roja generaron también su mística, apropiándose de rasgos cristianos (el símbolo evidente de la cruz), con principios de abnegación y misericordia. La privatización de la ayuda, enmarcada en una institución de estas características, les permitía a sus miembros visualizarse como un elemento de seguridad y orden científico: con sus transportes modernos y enfermeras ataviadas con impolutas cofias blancas, la Cruz Roja podía demostrar la calma y suficiencia de una perfecta organización supranacional.

Finalmente, los afanes nacionalistas de mediados de la década de 1930 debieron impactar en los participantes de las conferencias. Algunos se limitaron a señalar que el amor a la patria se podía muy bien ajustar al de toda la humanidad, pero otros se inclinaron por operativas necesarias, visualizando ya un futuro contexto bélico. Tal cuestión involucraba diferentes actores (y no solo a las Fuerzas Armadas), porque les permitía entrever que, si no hubiese más remedio, sería este un proceso organizado atento a principios y reglas, con el ejercicio de una "caballerosidad" proveniente de los orígenes mismos de la asociación y así, salvar al mundo de la pérdida de la civilización. Hacia 1940, cuando se realizó en Santiago de Chile el Cuarto Congreso Panamericano de la Cruz Roja, el contexto internacional era diferente ya que la Segunda Guerra Mundial impuso otras presiones a los países latinoamericanos. La preparación para la atención de los heridos y la situación de los prisioneros volvieron a estar en la mira de los objetivos de las sociedades nacionales de la Cruz Roja americanas, quienes habían sido persuadidas en las décadas de 1920 y 1930 para actuar a favor de la atención social en tiempos de paz y con una agenda vinculada sobre todo a la salud.

\section{AGRADECIMIENTOS}

Agradecemos parte de la información brindada al Dr. Jon Arrizabalaga, del Consejo Superior de Investigaciones Científicas (CSIC) de Barcelona. Este artículo forma parte del proyecto de investigación
“Burocracias e instituciones en la Argentina: salud, educación, tecnología, defensa y administración en contexto histórico (siglo XX)", aprobado por Resolución Nº 044/17 Consejo Directivo, Facultad de Ciencias Humanas, Universidad Nacional de La Pampa. 


\section{REFERENCIAS BIBLIOGRÁFICAS}

1. The Meetings of the International Red Cross in Prague. International Review of the Red Cross. 1961;1(8):415-422.

2. Meyer M. The importance of the International Conference of the Red Cross and Red Crescent to National Societies: fundamental in theory and in practice. International Review of the Red Cross. 2009;91(876):713-732.

3. Dunant H. Recuerdo de Solferino. Madrid: Comité Internacional de la Cruz Roja; 1982.

4. Muñoz Blanco ME. Cruz Roja Española: Un estatuto jurídico singular. Madrid: Tecnos; 1999.

5 García Reyes JC, Arrizabalaga J. Comunicación científica e innovación tecnológica en la primera Cruz Roja, 1863-1876. História, Ciência, SaúdeManguinhos. 2016;20(2):847-865.

6. Hutchsinson J. Champions of charity: War and the rise of the Red Cross. Boulder: Westview Press; 1996.

7. Feuerhahn W, Rabault-Feuerhahn P. Présentation: La science à l'echelle internationale. Revue Germanique Internationale. 2010;(12):5-15.

8. Long JD. Relación de la Oficina Sanitaria Panamericana con la Liga de las Sociedades de la Cruz Roja. Boletín de la Oficina Sanitaria Panamericana. 1924;3(1):10-15.

9. Cueto M, Palmer S. Medicina e Saúde Pública na América Latina: Uma História. Río de Janeiro: Fiocruz; 2016.

10. Cueto M. El valor de la salud: Historia de la Organización Panamericana de la Salud. Washington: OPS; 2004.

11. The League of Red Cross Societies. American Journal of Public Health. 1921;11(11):985-990.

12. Cueto M. (ed.) Missionaries of Science: The Rockefeller Foundation and Latin America. Bloommington-Indianapolis: Indiana University Press; 1994.

13. Douki C, Minard P. Histoire globale, histoires connectées: un changement d'échelle historiographique? Revue d'histoire moderne et contemporaine. $2007 ; 5(54): 7-21$.

14. Biernat C, Cerdá JM, Ramacciotti K, (dir.). La salud pública y la enfermería en la Argentina. Bernal: Universidad Nacional de Quilmes; 2015.
15. Di Stefano R, Sábato H, Romero JL, Moreno JL. De las cofradías a las organizaciones de la sociedad civil: Historia de la iniciativa asociativa en Argentina, 1776-1990. Buenos Aires: Gadis; 2002.

16. Veronelli JC, Veronelli Correch M. Los orígenes de la salud pública en la Argentina. Tomo I. Buenos Aires: Organización Panamericana de la Salud; 2004.

17. Alvarez A, Di Liscia MS. Entre pujas y facciones: la Cruz Roja Argentina (1864-1914). Boletín del Instituto de Historia Argentina y Americana Dr. Emilio Ravignani. En prensa, 2020.

18. Acha O. Catolicismo social y feminidad en la década de 1930: de damas a mujeres. En: Acha $\mathrm{O}$, Halperin $\mathrm{P}$, (comp.). Cuerpos, géneros, identidades: Estudios de historia de género en $\mathrm{Ar}$ gentina. Buenos Aires: Ediciones del Signo; 2000. p. $195-228$.

19. Una mujer de Estado. Revista de la Cruz Roja Argentina. 1923;1(7-8):25-26.

20. Guy D. Las mujeres y la construcción del Estado de Bienestar: Caridad y creación de derechos en Argentina. Buenos Aires: Prometeo Libros; 2011.

21. Ciafardo EO. Las damas de Beneficencia y la participación social de la mujer en la ciudad de Buenos Aires, 1880-1920. Anuario IEHS. 1990;(5):161-170.

22. Pita V. Cabellos largos, cerebro corto: Las difíciles relaciones entre las mujeres de la Sociedad de Beneficencia de la Capital y los médicos porteños, 1880-1905. En: Bravo MC, Gil Lozano F, Pita VS. Historias de luchas, resistencias y representaciones: Mujeres en la Argentina, siglos XIX y XX. San Miguel de Tucumán: Edunt; 2007. p. 15-49.

23. Anuncio. Revista de la Cruz Roja Argentina. 1923;1(7-8):36.

24. La solemne sesión de apertura de la Conferencia. Revista de la Cruz Roja Argentina. $1923 ; 1(7-8)$

25. Lozano N. El libro de la Cruz Roja Argentina. Buenos Aires: Talleres Gráficos de la Compañía General Fabril Financiera; 1932.

26. Eilers K. René Sand (1877-1953) and his contribution to International Social Work, IASSWPresident 1946-1953. Social Work and Society International Online Journal. 2007;5(1).

27. Programa de agasajos y festejos. Revista de la Cruz Roja Argentina. 1923;1(7-8). 
28. Liernur J.F. Arquitectura en la Argentina del siglo XX: La construcción de la modernidad. Buenos Aires: Fondo nacional de las Artes; 2001.

29. Comisiones de la Conferencia. Revista de la Cruz Roja Argentina. 1923;1(7-8).

30. Roosen MR. Memoria presentada ante la I Conferencia Panamericana de las Cruces Rojas en Buenos Aires. Uruguay; 1923. Localizado en: Archivo de la Biblioteca de la Facultad de Medicina, Universidad de Buenos Aires.

31. La exposición de Higiene. Revista de la Cruz Roja Argentina. 1923;1(7-8).

32. The World's Health. Revista mensual publicada por la Liga de Sociedades de la Cruz Roja. 1924;5(2).

33. Sand R. Organización internacional de la higiene y obras humanitarias: Discurso pronunciado en la conferencia para eliminar las causas de la guerra. Revista de la Cruz Roja Argentina. 1927;5(49).

34. The World's Health. Revista mensual publicada por la Liga de Sociedades de la Cruz Roja. 1926;7(9).

35. Trejo C. La obra de la Cruz Roja Argentina en sus últimos cinco años. Revista de la Cruz Roja Argentina. 1925;3(29-30):29-35.

36. Thorp R. Progreso, pobreza y exclusión: una historia económica de América Latina en el siglo XX. Washington: Banco Interamericano de Desarrollo, Unión Europea; 1998.

37. Salvatore R. Imágenes de un imperio: Estados Unidos y las formas de representación de América Latina. Buenos Aires: Sudamericana; 2006.

38. Conferencia Panamericana. Revista de la Cruz Roja Argentina. 1926;4(33-34).

39. Coolidge C. Speeches as President (19231929): Address to the Annual Convention of the American Red Cross [Internet]. 1926 [citado 9 dic 2018]. Disponible en: https://tinyurl.com/y2ttjfad

40. Cruz Roja Argentina. Su programa en épocas de paz: Llamado al pueblo. Buenos Aires: Imprenta Estrach; 1919.

41. Autoridades. Revista de la Cruz Roja Argentina. 1926;4(35).

42. Asamblea General Ordinaria. Revista de la Cruz Roja Argentina. 1926;4(37).
43. Información nacional. El Monitor de Educación Común. 1924;42(31):107-109.

44. La delegación argentina a la Conferencia de Washington. Revista de la Cruz Roja Argentina. 1926;4(38).

45. La Segunda Conferencia Panamericana. Revista de la Cruz Roja Argentina.1926;4(38).

46. Segunda Conferencia Panamericana de la Cruz Roja. Boletín de la Oficina Sanitaria Panamericana. 1926;5(8):347-350.

47. Lozano N. Acción social de la Cruz Roja. Revista de la Cruz Roja Argentina. 1926;4(42).

48. Cruz Vermelha Brasileira. Terceira Conferencia Pan-Americana da Cruz Vermelha, Rio de Janeiro, 15-26 de Setembro de 1935: Relatório. Rio de Janeiro: Cruz Vermelha Brasileira; 1936.

49. Drinot $P$. Introduction. En: Drinot $P$, Knight A, (ed.). The Great Depression in Latin America. London: Duke University Press; 2014.

50. La Cruz Roja en 1934. Revista de la Cruz Roja Argentina. 1935;13(140).

51. Larrosa JAR. La Tercera Conferencia Panamericana. Revista de la Cruz Roja Argentina. 1935;13 (141).

52. Actividad de la Sociedad Nacional de la Cruz Roja en el marco de los Convenios de Ginebra. Tercera Conferencia Panamericana, Río de Janeiro: sept. 15-26 de 1935. Comité Internacional de la Cruz Roja; 1935. Localizado en: Archivo de la Biblioteca de la Facultad de Medicina, Universidad de Buenos Aires.

53. Tercera Conferencia Panamericana de la Cruz Roja, del 15 al 25 de septiembre de 1935. Revista de la Cruz Roja Argentina. 1935;13(146).

54. Porras Gallo MI, Ballester Añón R. Luces y sombras de la erradicación de las enfermedades infecciosas: Interés historiográfico y reflexión para la salud pública actual. En: Porras Gallo MI, Báguena Cervellera MJ, Ayarzagüena Sanz M, Martín Espinosa NM, Ballester Añón R, Beldarraín Chaple $E$, et al. (coords.). La erradicación y el control de las enfermedades infecciosas. Madrid: Catarata; 2016.

55. Dalla Corte Caballero G. La guerra del Chaco: Ciudadanía, Estado, Nación en el siglo XX. La crónica fotográfica de Carlos de Sanctis. Rosario: Prohistoria Ediciones; 2010.

56. Actividad de la Sociedad Nacional. En: III Conferencia Panamericana. Buenos Aires: Cruz Roja; 1935. 
57. Tercera Conferencia Panamericana. Revista de la Cruz Roja Argentina. 1935;13(146).

58. Bendita seas. Revista de la Cruz Roja Argentina. 1935;13(146).

59. Scharagrodsky PD. El scautismo en la educación física bonaerense argentina, o acerca del buen encauzamiento varonil (1914-1916). Revista Brasileira de Ciências do Esporte. 2008;29(3):155171.

60. Di Liscia MS. Del brazo civilizador a la defensa nacional: Políticas sanitarias, atención médica y población rural (Argentina, 1900-1930). Historia Caribe. 2017;12(31):159-193.

\section{FORMA DE CITAR}

Di Liscia MS, Alvarez AC. La Cruz Roja, el panamericanismo y la salud en el período de entreguerras. Salud Colectiva. 2019;15:e2116. doi: 10.18294/sc.2019.2116.

Recibido: 11 de diciembre de 2018 | Versión final: 31 de agosto de 2019 | Aprobado: 11 de septiembre de 2019

Esta obra está bajo una licencia de Creative Commons Reconocimiento-NoComercial 4.0

Internacional. Reconocimiento - Permite copiar, distribuir y comunicar públicamente la obra.

A cambio, se debe reconocer y citar al autor original. No Comercial - Esta obra no puede ser

utilizada con finalidades comerciales, a menos que se obtenga el permiso.

http://dx.doi.org/10.18294/sc.2019.2116 